\title{
Efeito da Administração Crônica de Extrato Aquoso de Rubus rosifolius sobre o Perfil Glicêmico e Ponderal de Ratos Induzidos ao Diabetes e Obesidade
}

Effect of Chronic Administration of Aqueous Extract of Rubus rosifolius on Glycemic and Weight Profile of Mice Induced Hyperglycemia and Obesity

\section{Gabriela Iyzuka Gullo', Cleber Wagner de Carvalho ${ }^{1}$, Gislene Ferreira².}

1. Acadêmicos do $6^{\circ}$ ano do Curso de Medicina da Faculdade de Medicina de Itajubá (FMIt). Itajubá/MG

2. Nutricionista e Mestre em Microbiologia pela Universidade federal de Viçosa. (UFV). Professora da Faculdade de Medicina de Itajubá (FMIt). Itajubá/MG

Instituição: Faculdade de Medicina de Itajubá (FMIt). Itajubá/MG

Recebido em janeiro de 2015

Aceito em março de 2015

\section{Correspondência:}

Gabriela Iyzuka Gullo

Av. Renó Junior, 368 São Vicente

Itajubá/MG

CEP: $37502-138$

Fone: (35) 91433520

E-mail: gabiigullo@hotmail.com

\section{RESUMO}

Objetivo: investigar os efeitos do uso crônico de Rubus rosifolius sobre o perfil glicêmico e ponderal de ratos induzidos à hiperglicemia e obesidade. Metodologia: Foram utilizados 32 ratos machos, recém-nascidos, da linhagem Wistar, que foram divididos em 5 grupos $(\mathrm{n}=8)$ : $\mathrm{C} 1$ - Controle $\mathrm{NaCl}$; C2 - Controle Glutamato; T1 - Tratamento NaCl Curto prazo; T2 Tratamento NaCl Longo prazo; T3 - Tratamento Glutamato Longo prazo. Os Grupos C1, T1 e T2 não foram induzidos ao diabetes, recebendo solução salina $(\mathrm{NaCl}$ 0,9\%). Os Grupos C2 e T3, receberam injeção subcutânea de glutamato monossódico por 4 dias após o segundo dia de vida para indução do diabetes e obesidade. Após o período de desmame (30 dias), todos os grupos receberam ração da marca Purina ${ }^{\circledR}$ ad libitum. Os Grupos C1 e C2 receberam apenas água durante todo o experimento, os grupos T2 e T3 receberam o extrato aquoso de Rubus rosifolius durante todo o experimento, o grupo T2 recebeu o extrato da folha apenas nos últimos 15 dias de experimento. Os tratamentos foram conduzidos por 45 dias. Resultados: Houve uma diminuição altamente significante no Índice de Lee do grupo T3 em comparação ao grupo C2. Todavia, não houve variações estatisticamente significativas nos índices glicêmicos entre os diferentes grupos. Conclusão: A propriedade hipoglicemiante do extrato de Rubus rosifolius não pode ser comprovada através desde estudo, mas houve uma diminuição altamente significativa no Índice de Lee no grupo tratado, sugerindo um provável efeito no desenvolvimento da massa desses animais.

Palavras-chave: Rubus rosifolius, hiperglicemia, obesidade.

\begin{abstract}
Objective: To investigate the effects of chronic use of Rubus rosifolius on glycemic and weight profile of rats induced to hyperglycemia and obesity. Methods: 32 rats were used, newborns, Wistar, which were divided into 5 groups $(\mathrm{n}=8)$ : $\mathrm{C} 1$ - Control $\mathrm{NaCl} ; \mathrm{C} 2$ - Control glutamate; T1 - Treatment $\mathrm{NaCl}$ Short term; T2 - Long-term treatment NaCl; T3 - Glutamate Longterm treatment. Groups $\mathrm{C} 1, \mathrm{~T} 1$ and $\mathrm{T} 2$ were not induced to diabetes, receiving saline $(0.9 \% \mathrm{NaCl}) . \mathrm{C} 2$ and $\mathrm{T} 3$ groups received subcutaneous injection of monosodium glutamate for 4 days after the second day of life for the induction of diabetes and obesity. After a weaning period (30 days), all groups received feed Purina brand ad libitum. The $\mathrm{C} 1$ and $\mathrm{C} 2$ groups received only water throughout the experiment, the T2 and T3 groups received aqueous extract of Rubus rosifolius throughout the experiment, the T2 group received the leaf extract only in the last 15 days of the experiment. The treatments were conducted for 45 days. Results: There was a highly significant decrease in the T3 group Lee index compared to the C2 group. However, there was no statistically significant changes in glycemic indices between different groups. Conclusion: The hypoglycemic property Rubus rosifolius extract can not be proven through long study, but there was a highly significant decrease in the Lee index in the treated group, suggesting a likely effect on the development of the mass of these animals
\end{abstract}

Keywords: Rubus rosifolius, hyperglycemia, obesity. 


\section{INTRODUÇÃO}

O diabetes mellitus é um grupo de distúrbios metabólicos caracterizados pela elevação da glicemia em jejum. A hiperglicemia resulta de defeitos na secreção da insulina, causando sua deficiência absoluta ou relativa, na ação da insulina, ou mais frequentemente, em ambas. ${ }^{1,2}$ A expressão clínica do diabetes é representada por alterações metabólicas que podem associar-se a complicações neuropáticas e vasculares. As modificações metabólicas incluem, além da hiperglicemia, alterações no metabolismo de proteínas e lipídios. O componente vascular compreende a macroangiopatia diabética, caracterizada pela maior frequência, gravidade e precocidade da aterosclerose do que em não diabéticos, e a microangiopatia diabética, a qual atinge em especial, os rins e a retina. A neuropatia diabética afeta especialmente os nervos periféricos, determinando polineuropatias periféricas sensora e motora. Por estas razões, o diabetes é a principal causa de cegueira e amputações não traumáticas em membros inferiores no adulto e de doença renal em estágio terminal. ${ }^{3-5}$

A maioria dos casos de diabetes pode ser classificada em dois grupos: tipo 1 ou tipo 2. O diabetes tipo 1 é um distúrbio autoimune caracterizado pela destruição das células $\beta$ e uma consequente deficiência absoluta de insulina. Responde por cerca de 5 a $10 \%$ de todos os casos, surgindo mais comumente antes dos 30 anos de idade. $\mathrm{O}$ diabetes tipo 2 resulta de uma associação de resistência periférica à ação da insulina e uma secreção inadequada das células $\beta$ pancreáticas, produzindo uma deficiência relativa de insulina. Representa $90 \%$ a $95 \%$ dos casos de diabetes, e em larga proporção, está ligada a obesidade. , 3,6 $^{2,3}$

Para o diagnóstico, pode-se valer da clínica, como poliúria, polidipsia, polifagia, acompanhados de fadiga, perda de peso e fraqueza, com detecção independente da última refeição, de nível plasmático elevado de glicose $(\geq 200 \mathrm{mg} / \mathrm{dL}){ }^{2}$ Os valores normais da glicose sanguínea são mantidos entre $70 \mathrm{e}$ 99mg/dL. Se o indivíduo estiver em jejum entre 8 e 12 horas após a ultima ingesta calórica, o diagnóstico se dá com glicemia igual ou superior a $126 \mathrm{mg} / \mathrm{dL}$. Pode se utilizar também para confirmação, a obtenção de glicemia maior ou igual a $200 \mathrm{mg} / \mathrm{dL}$, duas horas após sobrecarga de 75 gramas de glicose via oral. ${ }^{3,6-9}$

$\mathrm{O}$ diabetes mellitus figura uma epidemia mundial, que hoje atinge 285 milhões de pessoas, e estima-se que em 2025, esse número se eleve para 380 milhões e em 2030, para 438 milhões. De acordo com o Diabetes Atlas 2012 Update da Federação Internacional de Diabetes (FID), nas Américas Central e do Sul, na população entre 20 e 79 anos de idade, mais de 26,4 milhões de pessoas têm diabetes, o que corresponde a 9,2\% dos adultos e avalia-se que esse valor aumente para 39,9 milhões em 2030. A FID estima que em 2012, no Brasil, existiam 13,4 milhões de 
diabéticos, o que corresponde a 10,28\% da população adulta no período, dos quais 6,14 milhões desconheciam o diagnóstico, e atribuindo ainda 129.226 mortes ao diabetes e um custo de 1031,44 dólares por paciente. De acordo com os Indicadores e Dados Básicos (IDB) de 2011, a prevalência de diabetes mellitus em 2009 no Brasil era de $9,5 \%$ e em 2010, a taxa de mortalidade específica era de 28,8 por 100.000 habitantes. A FID estima que em 2012 gastou-se em todo mundo cerca de 471,6 bilhões de dólares com o diabetes, e que este foi responsável por 4,8 milhões de morte. ${ }^{10} \mathrm{~A}$ incidência do diabetes está aumentando, principalmente no mundo em desenvolvimento, o que reflete a adoção de estilos de vida mais sedentários. Diante da elevada incidência e do alto custo aos cofres públicos, trata-se de um problema de saúde pública global, que exige medidas de controle e prevenção. ${ }^{11,12}$

O uso de infusões de plantas, oriundas do conhecimento popular, para o controle das doenças deve ser valorizado e constitui uma importante fonte para a descoberta de novos fármacos. Porém, o uso popular indiscriminado de plantas também oferece riscos, inclusive toxicidade, por isso a importância de estudos para comprovação terapêutica. A planta Rubus rosifolius, popularmente conhecida como amora silvestre, é empregada por conhecimento empírico para o controle glicêmico de diabéticos, sob a forma de infusão das folhas. A amora silvestre pertence à família Rosaceae, ao gênero Rubus e à espécie rosifolius e é um subarbusto terrícola, com ocorrência no Cerrado e Mata Atlântica, podendo atingir 1,0 a 1,5 metros de altura. ${ }^{13-17}$

A pesquisa da provável propriedade hipoglicemiante da Rubus rosifolius representa não apenas uma nova forma para o controle glicêmico de uma doença que se expande pelo mundo, como também uma alternativa econômica e sustentável viável a toda população. Comprovada sua eficiência, poderá tornarse um fitoterápico a integrar as Farmácias Vivas - locais onde se planta, manipula e dispensa gratuitamente fitos, prescritos por médicos locais, refletindo mais um aspecto benéfico da Política Nacional de Plantas Medicinais e Fitoterápicos. ${ }^{18}$

O objetivo do presente trabalho foi investigar os efeitos do uso crônico de Rubus rosifolius sobre o perfil glicêmico e ponderal de ratos induzidos à hiperglicemia e obesidade.

\section{MATERIAIS E MÉTODOS}

A pesquisa foi realizada na Faculdade de Medicina de Itajubá (FMIt), nas salas de Experimentação Animal do Biotério e nos Laboratórios de Bioquímica e Patologia. Os experimentos obedeceram às normas práticas didáticas-científicas da Lei Federal 11.794 e às orientações do Colégio Brasileiro de Experimentação Animal (COBEA) e foi aprovado pelo CEUA, sob o protocolo número 01/13. 
Para o estudo, foram utilizados 32 ratos machos, recém-nascidos, da linhagem Wistar, provenientes do biotério da Faculdade de Medicina de Itajubá. A determinação dessa amostra foi obtida através da análise de trabalhos referidos na literatura, que estudaram o efeito da administração oral do extrato de Baccharis dracunculifolia em 32 ratos machos da linhagem Wistar por 30 dias. Outro estudo avaliou o efeito da suplementação de vitamina $\mathrm{C}$ em 32 ratos diabéticos em 90 dias, contribuindo também para a determinação da amostra. ${ }^{19-21}$

Os animais foram divididos aleatoriamente em 05 grupos de 8 ratos $(\mathrm{n}=$ 8), sendo denominados: Grupo Controle $\mathrm{NaCl}$ (C1), Grupo Tratamento Curto Prazo $\mathrm{NaCl}$ (T1), Grupo Tratamento Longo Prazo $\mathrm{NaCl}$ (T2), Grupo Controle Glutamato (C2) e Grupo Tratamento Longo Prazo Glutamato (T3).

Para a indução ao diabetes e à obesidade, foi administrado glutamato monossódico na concentração de $4 \mathrm{mg} / \mathrm{g}$ de peso corporal nos grupos C2 e T3, via subcutânea, por quatro dias consecutivos a partir do segundo dia de vida. ${ }^{19,22,23}$

Devido às suspeitas de que o estresse causado pela manipulação possa modular o desenvolvimento de diabetes nos animais, ${ }^{24}$ os ratos dos grupos $\mathrm{C} 1, \mathrm{~T} 1 \mathrm{e} \mathrm{T} 2$ receberam injeção subcutânea de solução salina $0,9 \%(\mathrm{NaCl})$, a fim de submeter todos os animais do experimento ao mesmo tipo de estresse.
Até $0 \quad 30^{\circ}$ dia, os animais receberam apenas amamentação materna. Após esse período, foram divididos em 5 grupos $(n=8)$ e mantidos em gaiolas plásticas com ciclo claro-escuro de 12 horas, com livre acesso à água/extrato e à ração comercial da marca Purina ${ }^{\circledR} a d$ libitum.

Sendo assim, estratificaram-se os animais nos seguintes grupos:

- Grupo Controle $\mathrm{NaCl}(\mathrm{C} 1)$ - Injeção subcutânea $\mathrm{NaCl}$ 0,9\%, durante quatro dias, a partir do segundo dia do experimento, mantidos com ração e água durante todo o estudo.

- Grupo Tratamento Curto Prazo $\mathrm{NaCl}$ (T1) - Injeção subcutânea $\mathrm{NaCl}$ 0,9\%, durante quatro dias, a partir do segundo dia do experimento, mantidos com ração durante todo o experimento e extrato aquoso de Rubus rosifolius durante os últimos 15 dias de experimento.

- Grupo Tratamento Longo Prazo $\mathrm{NaCl}$ (T2) - Injeção subcutânea $\mathrm{NaCl}$ 0,9\%, durante quatro dias, a partir do segundo dia do experimento, mantidos com ração e extrato aquoso de Rubus rosifolius durante todo o estudo.

- Grupo Controle Glutamato (C2) Injeção subcutânea de Glutamato Monossódico, durante quatro dias, a partir do segundo dia do experimento, mantidos com ração e água durante todo o estudo.

- Grupo Tratamento Longo Prazo Glutamato (T3) - Injeção subcutânea de Glutamato Monossódico, durante quatro dias, a partir do segundo dia do experimento, mantidos com ração e extrato aquoso de Rubus rosifolius durante todo o estudo.

O extrato aquoso foi preparado na concentração de $50 \mathrm{~g}$ de folhas secas de 
Rubus rosifolius para $1000 \mathrm{~mL}$ de água. Esta concentração foi obtida com base no conhecimento popular, uma vez que não há estudos na literatura sobre a propriedade hipoglicemiante da folha de Rubus rosifolius. As folhas de Rubus rosifolius foram extraídas quinzenalmente, ao longo do experimento, na zona rural do município de São Luiz do Paraitinga, no estado de São Paulo.

No último dia do período experimental $\left(105^{\circ}\right.$ dia de vida dos animais), respeitando-se o período de 8 horas de jejum, os animais foram anestesiados com Ketamina $(50 \mathrm{mg} / \mathrm{Kg}) /$ Xylazina $(25 \mathrm{mg} / \mathrm{Kg})$ por via intramuscular e submetidos à punção aspirativa do ventrículo esquerdo, produzindo significativa hipovolemia e consequente eutanásia. As amostras de sangue coletadas foram centrifugadas em centrífuga clínica (Excelsa, Fanen) a 2000 rpm, e o soros resultantes $(1,5 \mathrm{~mL} /$ rato $)$ armazenados em eppendorf e congelados a $-4^{\circ} \mathrm{C}$, para futuras dosagens laboratoriais.

A glicemia foi determinada por espectrofotometria, utilizando $\mathrm{o}$ espectrofotômetro da marca Quimis (modelo Q-108 DRD), dosado pelos Kits enzimáticos Labtest - Glicose PAP Liquiform. O Índice de Lee foi calculado baseado nas medidas de massa e comprimento dos animais após a anestesia para punção endocárdica.

Para a análise estatística, foi utilizado o Software BioEstat 5.0 ®, em que o teste $\mathrm{t}$ de Student comparou as variâncias estatísticas t e $p$, através de dois grupos independentes em cada momento. Considerou-se uma estatística significativa os casos em que $\mathrm{p}$ foi menor que 0,05 . Quando $p$ situou-se entre 0,05 e 0,10 foi referido provável significância, em que p é a probabilidade de erroneamente concluir pela significância. ${ }^{26}$

\section{RESULTADOS}

Os valores absolutos em médias aritméticas e seus respectivos desviospadrões da taxa de glicemia e Índice de Lee dos diferentes grupos do experimento, assim como os valores de $\mathrm{p}$ da comparação estatística entre os grupos, podem ser observados nas Tabelas 1, 2 e 3.

A análise dos Índices de Lee revelou um aumento em valores absolutos desse parâmetro no grupo $\mathrm{C} 2$ em relação ao grupo $\mathrm{C} 1$, mas não foi estatisticamente significante $(\mathrm{p}=0,4453)$. (Tabelas 2 e 3$)$. $\mathrm{Na}$ comparação entre os grupos C1 e T1, notase, em valores absolutos do Índice de Lee, um aumento muito discreto em $\mathrm{T} 1$ comparado a $\mathrm{C} 1$, porém de forma não significativa estatisticamente $(\mathrm{p}=0,8652)$. Na relação entre os grupos $\mathrm{C} 1$ e T2, houve discreta diminuição no valor absoluto do Índice de Lee no grupo T2, não sendo significativo em âmbito estatístico ( $\mathrm{p}=0,3102)$; no entanto, no grupo T3 houve uma diminuição altamente significante no Índice de Lee, quando comparado ao grupo C2 ( $\mathrm{p}=0,0064)$, o que se evidencia pela análise dos valores absolutos desse 
parâmetro nos dois grupos (Tabelas 1 e 3 e Gráfico 1).

O valor absoluto do Índice de Lee embora tenha sido menor no grupo T2 ao compara-lo com o grupo T1, não se sucedeu de forma significante estatisticamente $(p=$
0,2482). Na comparação dos grupos tratados por longo prazo T2 e T3, ainda que tenha ocorrido uma diminuição mais expressiva no valor absoluto do Índice de Lee em T3, não ocorreu de forma significante $(\mathrm{p}=0,1130)$.

Tabela 1 - Médias e Desvios Padrões das Massas, Comprimentos e Índice de Lee dos animais dos grupos Controle $\mathrm{NaCl}(\mathrm{C} 1$ ), Tratamento Curto Prazo $\mathrm{NaCl}$ (T1), Tratamento Longo Prazo $\mathrm{NaCl}$ (T2), Controle Glutamato (C2) e Tratamento Longo Prazo Glutamato (T3)

\begin{tabular}{cccc}
\hline Grupos & Comprimento $(\mathbf{c m})$ & Massa $(\mathbf{g})$ & Índice de LEE \\
\hline C1 & $26.00 \pm 1,41$ & $377.5 \pm 21,31$ & $278,32 \pm 10,95$ \\
T1 & $25.75 \pm 1,25$ & $372.5 \pm 20,68$ & $279,76 \pm 11,96$ \\
T2 & $26.40 \pm 1,14$ & $369.2 \pm 22.56$ & $271,87 \pm 6,73$ \\
C2 & $23.75 \pm 1,25$ & $308.0 \pm 30.24$ & $284,45 \pm 10,26$ \\
T3 & $25.60 \pm 0,89$ & $316.0 \pm 34.29$ & $265,81 \pm 3,54$ \\
\hline
\end{tabular}

Comparando-se os valores absolutos entre os grupos controles $\mathrm{C} 1$ e $\mathrm{C} 2$ e controle $\mathrm{C} 1$ e tratamento $\mathrm{T} 1$, observou-se um aumento na glicemia dos grupos $\mathrm{C} 2 \mathrm{e}$ T1; porém, trata-se de aumentos que não são estatisticamente significantes, com $\mathrm{p}=0,6274$ e $\mathrm{p}=0,2937$, respectivamente. Da mesma forma, entre os grupos $\mathrm{C} 1$ e $\mathrm{T} 2$, embora tenha havido um aumento no valor absoluto da glicemia neste último grupo, os valores não foram estatisticamente significantes $\quad(p=0,7510) . \quad O$ grupo $T 3$ apresentou um aumento no valor absoluto de glicemia em relação ao grupo $\mathrm{C} 2$, também não significativo $(\mathrm{p}=0,7209)$.
Entre os grupos tratamentos $\mathrm{T} 1 \mathrm{e}$ $\mathrm{T} 2$, observou-se em valores absolutos uma diminuição da glicemia no grupo de tratamento por longo prazo (T2), porém trata-se de uma diminuição não significativa estatisticamente $(\mathrm{p}=0,3805)$. Os grupos tratamentos por longo prazo $\mathrm{T} 2 \mathrm{e}$ T3 revelaram um aumento nos índices glicêmicos nesse último grupo, mas a estatística demonstrou tratar-se de um aumento não significativo $(\mathrm{p}=0,6856)$. (Tabelas 2 e 3). 
Tabela 2 - Médias e Desvios Padrões das Glicemias dos animais dos grupos Controle $\mathrm{NaCl}$ (C1), Tratamento Curto Prazo NaCl (T1), Tratamento Longo Prazo NaCl (T2), Controle Glutamato (C2) e Tratamento Longo Prazo Glutamato (T3)

\begin{tabular}{cc}
\hline Grupos & Glicemia $(\mathbf{m g} / \mathbf{d L})$ \\
\hline $\mathrm{C} 1$ & $157.82 \pm 53,92$ \\
$\mathrm{~T} 1$ & $195.70 \pm 37,81$ \\
$\mathrm{~T} 2$ & $168.82 \pm 46,22$ \\
$\mathrm{C} 2$ & $172.69 \pm 21,83$ \\
$\mathrm{~T} 3$ & $178.82 \pm 26,42$ \\
\hline
\end{tabular}

Tabela 3 - Valores de p nas comparações de Glicemia e Índice de Lee dos grupos Controle $\mathrm{NaCl}$ (C1), Tratamento Curto Prazo $\mathrm{NaCl}$ (T1), Tratamento Longo Prazo $\mathrm{NaCl}$ (T2), Controle Glutamato (C2) e Tratamento Longo Prazo Glutamato (T3)

\begin{tabular}{ccc}
\hline Parâmetros & Glicemia & Índice de Lee \\
\hline $\mathbf{p}(\mathbf{C 1} / \mathbf{C 2})$ & 0.6274 & 0.4453 \\
$\mathbf{p}(\mathbf{C} 1 / \mathbf{T 1})$ & 0.2937 & 0.8652 \\
$\mathbf{p}(\mathbf{C 1} / \mathbf{T} 2)$ & 0.7510 & 0.3102 \\
$\mathbf{p}(\mathbf{C 2} / \mathbf{T 3})$ & 0.7209 & 0.0064 \\
$\mathbf{p}(\mathbf{T 1} / \mathbf{T} 2)$ & 0.3805 & 0.2482 \\
$\mathbf{p}(\mathbf{T} 2 / \mathbf{T 3})$ & 0.6856 & 0.1130 \\
\hline
\end{tabular}

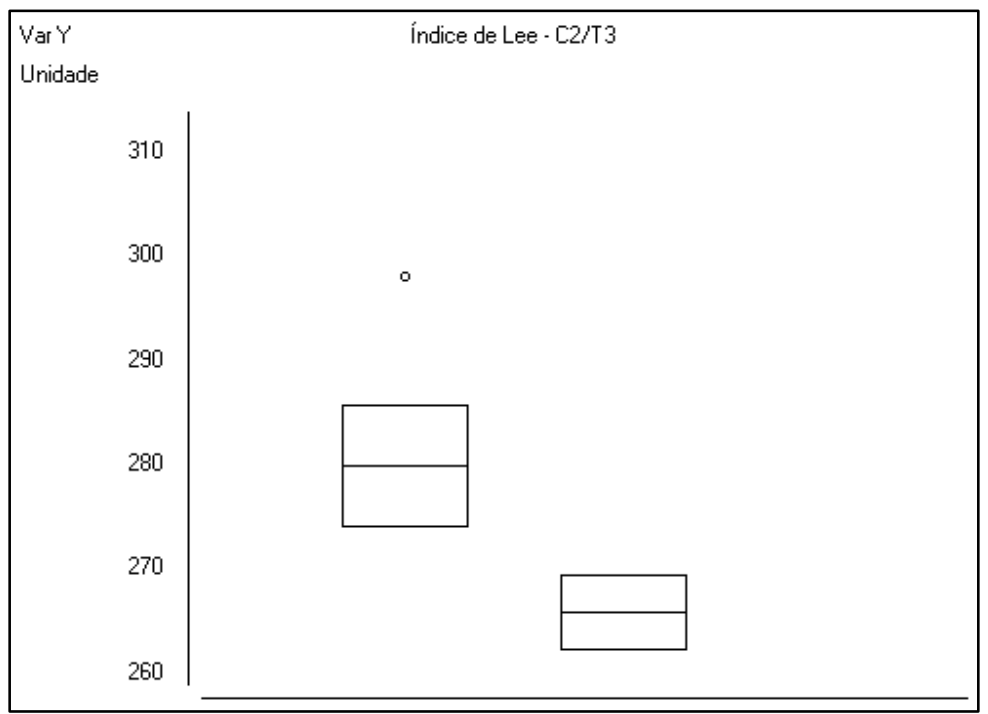

Gráfico 1 - Valores médios de Índice de Lee dos grupos Controle Glutamato (C2) e Tratamento Longo Prazo Glutamato (T3) $* \mathrm{p}<0,01$ 


\section{DISCUSSÃO}

A indução a hiperglicemia com injeções subcutâneas de glutamato monossódico nos animais observado em alguns estudos, não foi observada nessa pesquisa. A indução à obesidade também não foi constatada. Porém, em valores absolutos, observou-se um aumento da glicemia e do peso no grupo induzido com glutamato monossódico, em comparação com o grupo que recebeu apenas $\mathrm{NaCl}$.

Estatisticamente, como não houve diferença significativa nos valores de glicemia entre os grupos, a constatação da propriedade hipoglicemiante da planta Rubus rosifolius, demonstrada por Cordova et al, ficou prejudicada nesse estudo. Comparando-se, em valores absolutos, o grupo Controle Glutamato (C2) apresentou maiores taxa de glicemia do que o grupo Controle $\mathrm{NaCl}(\mathrm{C} 1){ }^{27}$

Analisando somente os grupos que não foram induzidos com glutamato monossódico, o grupo Controle $\mathrm{NaCl}$ (C1) apresentou os menores valores de glicemia, quando comparado aos grupos que receberam o extrato da planta, ao contrário do resultado esperado. Comparando os grupos T1 e T2, conclui-se que a realização do tratamento em longo prazo apresenta menor taxa glicêmica, podendo sugerir ser mais efetivo do que um tratamento em curto prazo.

Como a indução à hiperglicemia e à obesidade não foram conclusivas nesse estudo, não é relevante a comparação entre o Controle Glutamato (C2) e o Tratamento Longo Prazo Glutamato (T3).

Apesar da planta apresentar altos índices de manganês, o qual, além de auxiliar na formação óssea ajuda a regular o nível glicêmico no organismo, não houve diferença significativa desses parâmetros entre os grupos que receberam o extrato aquoso da planta Rubus rosifolius e os que receberam apenas água.

Em relação ao índice de Lee, equivalente ao índice de massa corporal em humanos, houve diferença significativa entre os grupos induzidos (C2 e T3). Porém, essa diminuição do índice de Lee no grupo T3 pode não ser devida à diminuição da massa, não podendo então ser considerada uma planta que apresente propriedades para redução de massa corporal.

\section{CONCLUSÃO}

Os dados coletados não permitem afirmar que o uso da infusão das folhas de Rubus rosifolius tenha reduzido de forma significativa os níveis glicêmicos dos animais, ou seja, não evidenciaram uma propriedade hipoglicemiante da planta. Todavia, pode-se concluir que a administração crônica da infusão foi capaz de reduzir de forma significativa o Índice de Lee dos animais, o que corresponderia ao IMC nos seres humanos. 


\section{REFERÊNCIAS}

1. Champe PC, Harvey RA, Ferrier DR. Bioquímica ilustrada. $4^{\mathrm{a}}$ ed. Porto Alegre: Artmed; 2009. 528p.

2. Kumar V, Abbas AK, Fausto N, Aster JC. Robbins e Cotran, bases patológicas das doenças. $8^{\text {a }}$ ed. Rio de Janeiro: Elsevier; 2010. 1457p.

3. Borges DR, (org). Atualização terapêutica de Prado, Ramos e Valle: diagnóstico e tratamento - 2012/2013. $24^{\mathrm{a}}$ ed. São Paulo: Artes Médicas; 2012. 1990p.

4. Nelson DL, Cox MM. Lehninger Princípios de bioquímica. $4^{a}$ ed. São Paulo: Sarvier; 2006. 1202p.

5. Brasileiro Filho G. Bogliolo Patologia. $7^{a}$ ed. Rio de Janeiro: Guanabara Koogan; 2006. 1472p.

6. Gross JL, Silveiro SP, Camargo JL, Reichelt AJ, Azevedo MG. Diabetes melito: diagnóstico, classificação e avaliação do controle glicêmico. Arq Bras Endocrinol Metab. 2002;46(1):1626.

7. Oliveira GC. Perfil físico funcional dos indivíduos com diabetes mellitus tipo 1 e 2, do município de matinhos[Dissertação]. Curitiba: Universidade Federal do Paraná; 2012.

8. Maraschin JF, Murussi N, Witter V, Silveiro SP. Classificação do diabete melito. Arq Bra Cardiol. 2010;95(2):40-6.

9. American Diabetes Association - ADA. Standards of medical care in diabetes. Diabetes Care. 2011;34(Suppl 1):S1161.

10. Federação Internacional de Diabetes FID. Atualização 2012, Américas Central e do Sul[Internet]. 2012. [Acesso em: 2013 Jan 23]. Disponível em: http://www.idf.org/sites/default/files/S ACA_5E_Update_Country.pdf

11. Brasil. Ministério da Saúde - MS. Datasus: IDB 2011, Prevalência de diabetes melito[Internet]. 2011. [Acesso em: 2013 Jan 22]. Disponível em: http://tabnet.datasus.gov.br/cgi/tabnet.e xe?idb2011/g01.def

12. Brasil. Ministério da Saúde - MS. Datasus: IDB 2011, mortalidade específica por diabetes melito[Internet]. 2011. [Acesso em: 2013 Jan 22]. Disponível em: http://tabnet.datasus.gov.br/cgi/tabcgi.e xe?idb2011/c12.def

13. Jornal Livre. Para que serve a amora[Internet]. [Acesso em: 2013 Jan 22]. Disponível em: http://www.jornallivre.com.br/138676/p ara-que-serve-a-amora

14. Plantas medicinais e fitoterapia. Amora: benefícios e propriedades medicinais [Internet]. [Acesso em: 2013 Jan 24]. Disponível em: http://www.plantasmedicinaisefitoterapi a.com/plantas-medicinais-amora.html

15. Infoescola, navegando e aprendendo. Biologia, reino plantae, amora[Internet]. [Acesso em: $2013 \mathrm{Jan}$ 24]. Disponível em: http://www.infoescola.com/frutas/amor a/

16. Marques CV, Teixeira VA. Efeitos do extrato aquoso de Morus nigra L. (amora-preta) sobre níveis plasmáticos de colesterol total, HDL e glicose de ratos wistar[Internet]. [Acesso em: 2013 Jan 24]. 2009 Disponível em: http://www.ufpel.tche.br/cic/2009/cd/pd f/CB/CB_01065.pdf

17. Bianchini RS. Rosaceae in lista de espécies da flora do Brasil. Jardim Botânico do Rio de Janeiro[Internet]. 2012. [Acesso em: 2013 Jan 22]. Disponível em: http://floradobrasil.jbrj.gov.br/2012/FB 032510

18. Uechi L. Neomondo - Fitoterapia: A medicina sustentável [Internet]. 2007 [Acesso em: 2013 Jan 11]. Disponível em:

http://www.neomondo.org.br/index.php /saude/150-fitoterapia-a-medicinasustentavel.

19. Hocayen PAS. Efeito da administração oral do extrato de Baccharis dracunculifolia na obesidade induzida por glutamato monossódico (MSG)[Dissertação]. Ponta Grossa/PR: Universidade de Ponta Grossa; 2012.

20. Cortez AC, Benedito HG, Agreste FR, Clebis NK, Bombonato PP. Estudo 
histomorfométrico do baço de ratos wistar sábios e diabéticos suplementados ou não pela vitamina $\mathrm{C}$. Pesqui Vet Bras. 29(10):834-40.

21. White PAS. Efeito do extrato aquoso de Abajeru (Crhysobalanus icaso) sobre o peso corporal, adiposidade e sensibilidade à insulina de camundongos obesos [Dissertação]. Aracaju/SE: Universidade Federal de Sergipe; 2011.

22. Junqueira PHT, Meneghin PV, Silva A, Vieira AF, Silva SL, Baracho NCV. Desenvolvimento e caracterização de um modelo experimental de obesidade por injeção subcutânea de glutamato monossódico em ratos. Rev Cienc Saúde. 2011;1(3):22-32.

23. Gomes RM, Marques AS, Torrezan R, Scomparin DX, Mathias PCF, Rinaldi W. Efeito de um programa de exercício físico moderado em ratos de diferentes modelos de obesidade. Rev Educ Fis/UEM. 2012;23(2):285-94.

24. Kirsten VR. Caracterização do modelo experimental NOD (Nonobese diabetic) em ambiente convencional [Dissertação]. Porto Alegre/RS: Faculdade de Medicina da PUCRS; 2006.

25. Scnaider TB, Souza C. Aspectos éticos da experimentação animal. Rev Bras Anestesiol. 2003;53(2):278-85.

26. Arango HG. Bioestatistica: teórica e computacional: testes paramétricos. $3^{\text {a }}$ ed. Rio de Janeiro: Guanabara Koogan; 2009. 438p.

27. Cordova SM, Benfatti CS, Magina MDA, Guedes A, Cordova CMM. Análise da atividade antimicrobiana de extratos isolados de plantas nativas da flora brasileira frente a Mycoplasma arginini, M. hominis e Ureaplasma urealyticum. RBAC. 2010; 42(4):241-4.

Correspondência: Gabriela Iyzuka Gullo Av. Renó Junior, 368 São Vicente Itajubá/MG CEP: 37502-138

Fone: (35) 91433520 E-mail: gabiigullo@ hotmail.com 